\title{
Noise Suppressing Edge Enhancement Based on Genetic Algorithm Taking Into Account Complexity of Target Images Measured with Fractal Dimension
}

\author{
Kohei Arai ${ }^{1}$ \\ Graduate School of Science and Engineering \\ Saga University \\ Saga City, Japan
}

\begin{abstract}
Method for noise suppressing edge enhancement based on genetic algorithm taking into account complexity of target images measured with fractal dimension is proposed. Through experiments with satellite remote sensing imagery data with additive noise, it is found that the proposed method shows appropriate edge enhancing performance with suppressing the additive noise in accordance with complexity of target images. It is also found that the proposed method requires a small computer resources in comparison to the method based on Simulated Annealing: SA.
\end{abstract}

Keywords-edge enhancement; fractal dimension; genetic algorithm; simulated annealing; remote sensing satellite imagery

\section{INTRODUCTION}

There are many edge enhancement methods in the field of image processing and analysis. Differentiation of images is just one example of the methods ${ }^{1}$. Prewitz operator, Sobel operator $^{2}$, Laplace operator ${ }^{3}$, Un-Sharpe masking ${ }^{4}$, Canny filter ${ }^{5}$ etc. are typical edge enhancement operators. On the other hand, there are also many methods of smoothing filter ${ }^{6}$ for noise suppressions. Averaging filter ${ }^{7}$, Median filter ${ }^{8}$, Gaussian filter ${ }^{9}$, etc. are typical smoothing filter. Most of cases of image processing and analysis requires edge enhancement with noise suppressions. Therefore, these edge enhancements and smoothing filters are applied to target images simultaneously or sequentially [1].

The most appropriate edge enhance method depends on characteristics of the target images, in particular, complexity of the target images. For instance, simple images do not have many edges while there are many edges in complex images. Therefore, it would better to take into account image complexity in parameter estimations of edge enhancement methods. Fractal dimension is one of the complexity representation methods. Hausdorff dimension ${ }^{10}$, Rényi

\footnotetext{
${ }^{1}$ http://www.mis.med.akita-u.ac.jp/ kata/image/sobelprew.html

${ }^{2}$ http://en.wikipedia.org/wiki/Sobel_operator

${ }^{3}$ http://en.wikipedia.org/wiki/Discrete_Laplace_operator

${ }^{4}$ http://en.wikipedia.org/wiki/Unsharp_masking

${ }^{5}$ http://en.wikipedia.org/wiki/Canny_edge_detector

${ }^{6} \mathrm{http} / / / \mathrm{en}$.wikipedia.org/wiki/Smoothing

${ }^{7}$ http://homepages.inf.ed.ac.uk/rbf/HIPR2/mean.htm

${ }^{8} \mathrm{http}: / /$ en.wikipedia.org/wiki/Median_filter

${ }^{9}$ http://en.wikipedia.org/wiki/Gaussian_filter

${ }^{10} \mathrm{http} / / /$ en.wikipedia.org/wiki/Hausdorff_dimension
}

dimension $^{11}$, Packing dimension ${ }^{12}$ are typical theoretical fractal dimensions. However, Minkowski-Bouligand dimension $^{13}$, Correlation dimension ${ }^{14}$ are widely used to use because these are relatively easy to calculate [2]. Therefore, probabilistic auto-correlative dimension is for representation of complexity of the target images in the paper.

Finding the most appropriate edge enhancement method is a kind of optimization problem solving. Namely, edge enhancement method with the most appropriate parameter set is obtained by using optimization problem solving in accordance with input image characteristics. There are some methods for optimization problem solving, linear and nonlinear optimization to find global optimum or local minima. Only the Simulated Annealing: SA ensure finding global optimum solution while some other optimization methods, such as Steepest Descent Method: SDM, Conjugate Gradient Method: CGM (Newton Raphson method), Genetic Algorithm: GA, etc. find one of local minima solutions. SA requires huge computer resources while the other methods require not so large computer resources [3].

The edge enhancement method with suppressing noise is based on LOG filter which apply Gaussian filter first and then apply Un-Sharp Masking followed by, taking into account input image characteristics of complexity which is detected by probabilistic auto-correlative dimension. Based on GA with appropriate parameters of cross-over, mutation, fitness function is defined with the complexity and the ratio of power spectrum of input and output images. In prior to apply the proposed edge enhancement method, median filter is applied for elimination of isolated noise pixels.

Experiments with remote sensing satellite images are conducted to validate the proposed edge enhancement method. The results show that output edge enhanced images with the proposed method and that from the SA based method are almost similar. Meanwhile, computation resources required for the proposed GA based method is almost 1/53 comparing to

11

http://ja.wikipedia.org/wiki/\%E3\%83\%95\%E3\%83\%A9\%E3\%82\%AF\%E3\% 82\%BF\%Е3\%83\%AB\%E6\%AC\%A1\%E5\%85\%83\#.E3.83.AC.E3.83.8B.E3. 83.BC.E6.AC.A1.E5.85.83

12 http://en.wikipedia.org/wiki/Packing_dimension

${ }^{13} \mathrm{http}$ ///en.wikipedia.org/wiki/Box-counting_dimension

${ }^{14} \mathrm{http}$ //en.wikipedia.org/wiki/Correlation_dimension 
that for the SA based method. Therefore, the proposed method is efficiently and effectively useful in comparison to the SA based method.

The following section describes the proposed method followed by some experimental results. Then finally concluding remarks is described together with some discussions.

\section{PROPOSED METHOD}

\section{A. LOG Filter}

The proposed edge enhancement method is based on LOG filter which is defined as follows,

Firstly, Gaussian filter is applied to input images followed by Un-Sharp Masking. Gaussian filter is defined in equation (1).

$$
g(j, i)=\sum_{y=-n}^{n} \sum_{x=-n}^{n} \operatorname{gauss}(x, y) f(j+x, i+y)
$$

where $f(j, i)$ and $g(j, i)$ denotes input and ouput images, two dimensional gray level in direction of line, pixel coordinates, $j$ and $i$ ( $x$ and $y$ ). Meanwhile, $n$ denotes image pixel size in directions of line and pixel. On the other hand, $\operatorname{gauss}(x, y)$ is expressed in equation (2).

$\operatorname{gauss}(x, y)=\frac{\exp \left(-\frac{x^{2}+y^{2}}{2 \sigma^{2}}\right)}{2 \pi \sigma^{2}}$

Also, Un-Sharp masking is defined as equation (3).

$$
g(j, i)=f(j, i)-\nabla^{2} f(j, i)
$$

where $\nabla^{2}$ denotes Laplace operator which is second order of differentiation of equation (4).

$\nabla^{2} f(x, y)=\frac{\partial f^{2}(x, y)}{\partial x^{2}} \mathbf{j}+\frac{\partial \mathbf{f}^{2}(\mathbf{x}, \mathbf{y})}{\partial \mathbf{y}^{2}} \mathbf{i}$

Thus, LOG filter is defined as equation (5).

$$
h(j, i)=g(j, i)-\nabla^{2} g(j, i)
$$

\section{B. Genetic Algorithm Based Edge Enhancement}

Fitness function which represents edge information is very important for GA based edge enhancements. Edge enhancement implies high frequency component enhancement. Therefore, the ratio of frequency components of input and output images would be used for a fitness function as defined in equation (6).

$$
F_{I}=\frac{P_{I_{\text {original }}}}{P_{I_{\text {transformed }}}}
$$

where $\mathrm{P}^{*}$ denotes power spectrum while $I_{\text {original }}$ and

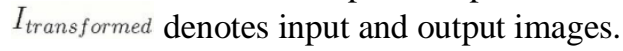

In the mean time, noise has to be suppressed as much as we can. Low pass filter is used for noise suppression. In general, noise spectrum is widely spreaded from zero to infinitive frequency components. Signal frequency component is degraded when the cut-off frequency of low pass filter is too low, noise can be suppressed greatly though. Therefore, a filter which allows to suppress higher frequency component maintaning edge components which are also higher frequency components is considered. The frequency has to be normalized because Nyquist frequency of images depends on image size. Therefore, normalized frequency is defined in equation (7).

$f=\frac{\sqrt{f_{x}^{2}+f_{y}^{2}}}{f_{N}}$

where $f_{N}$ denotes Nyquist frequency while $f_{x}$ and $f_{y}$ denotes frequency in direction of line and pixel. Furthermore, it would be better to normalize the frequency as follows,

when frequency is zero while Nyquist frequency is $f_{N}$, the normalized frequency is one. Therefore, the following weighting function is applied to frequency,

$w(f)=a \cdot \exp \left(f^{10}\right)+b$

where $w(0)=0, w(1)=1$ then

$\left\{\begin{array}{l}0.0=a \cdot \exp \left(0.0^{10}\right)+b \\ 1.0=a \cdot \exp \left(1.0^{10}\right)+b\end{array}\right.$

Therefore, a and $\mathrm{b}$ can be determined in equation (10).

$\left\{\begin{array}{r}a=1 /(e-1) \\ b=-1 /(e-1)\end{array}\right.$

Thus the equation (8) can be rewritten by equation (11).

$w(f)=\frac{\exp \left(f^{10}\right)-1}{e-1}$

As is mentioned before, fitness function is also normalized within the range of $[0, \sqrt{2}]$. Power spectrum $p\left(f_{I}\right)$ of the image $I$ is defined and also weighted sum of $p\left(f_{I}\right)$ can be defined in equation (12)

$P_{I}=\sum_{f_{I}=0}^{f_{N}} w\left(f_{I}\right) \cdot p\left(f_{I}\right)$

Thus fitness function of equation (6) is derived with the normalized and weighted sum of power spectrum, $\mathrm{P}^{*}\left(^{*}\right.$ denotes input $I_{\text {original }}$ and output images $\left.I_{\text {transformed }}\right)$. 


\section{Image Complexity}

Probabilistic auto-correlative dimension is used for representation of complexity of the target images. Let $F(a t)$ be a stochastic process as ${ }^{\forall} a>0$.

$$
F(a t)=a^{H} F(t)
$$

where $F(t)$ is a self-similar type of stochastic process with the power of $H$. The equation (13) implies that the probability density function of $F(a t)$ is identical to that of $a^{H} F(t)$. Thus probabilistic self-similar dimension $\mathrm{H}$ can be written in equation (14) through taking logarithmic function of expectation (E) maintaining $t$.

$H=\frac{-\log _{10}\left(\frac{E[F(a t)]}{E[F(t)]}\right)}{\log _{10}\left(\frac{1}{a}\right)}$

With the fixing $\mathrm{t}=1, \quad M\left(\frac{1}{n}\right)$ of surface division number of image grey-level of curved surface is defined as of image is devided into $\frac{1}{a} \times \frac{1}{a}$. In other word, the number of devision of $E[F(a)]$ with the unit length of $1 / a$ is defined as $Y\left(\frac{1}{n}\right)$. Taking logarithmic function of $N\left(\frac{1}{11}\right)$, equation (14) can be rewritten by equation (15).

$$
\log _{10} N(r)=-H \log _{10} r+\log _{10} N(1)
$$

where $r$ denotes $1 / a$. In general, $H$ is veraied depending on $r$. Edge components are maintained if the target image can be represented as fractal like images. Therefore, fractal dimension $\mathrm{H}$ can be determined through regressive analysis based on least square method with sample values of

$$
\left(\log _{10} r, \log _{10} N(r)\right),(r=1,2, \cdots)
$$

If the surface area of $S(r)$ can be written in equation (16),

$$
S(r)=r^{2} \cdot N(r)
$$

where the target image is divided into $r \times r$, then the equation (16) can be rewritten by equation (17)

$$
\log _{10} S(r)=(2-H) \log _{10} r+\log _{10} S(1)
$$

Thus fractal dimension can be calculated as estimation value of $2-H$ through regression analysis with

$$
\left(\log _{10} r, \log _{10} S(r)\right),(r=1,2, \cdots)
$$

based on least square method.

\section{Fitness Function of GA for Edge Enhancement}

Fractal dimension of grey-level images (half tone images) $D_{I}$ is within the range of 2 to $3,2 \leq D_{I}<3$. In order to normalize $D_{I}$ within the range of $[0,1], D_{I}-2$ is adopted as fitness function. Taking into accout the aforementioned normalized frequency components ratio, $\mathrm{Fi}$, the fitness function $f_{I}$ is defined as follows,

$f_{I}=\left(D_{I}-2\right) F_{I}$

Thus the edge enhancement method with suppressing noise is based on LOG filter which apply Gaussian filter first and then apply Un-Sharp Masking followed by, taking into account input image characteristics of complexity which is detected by probabilistic auto-correlative dimension is defined Based on GA with appropriate parameters of cross-over, mutation, fitness function is defined with the complexity and the ratio of power spectrum of input and output images. In prior to apply the proposed edge enhancement method, median filter is applied for elimination of isolated noise pixels.

\section{EXPERIMENTS}

\section{A. Data Used}

32 by 32 pixels of a portion of Landsat TM imagery data [4] of Saga city in Kyushu, Japan is used for the experiments. Original image is shown in Fig.1 together with frequency component in direction of line and pixel.

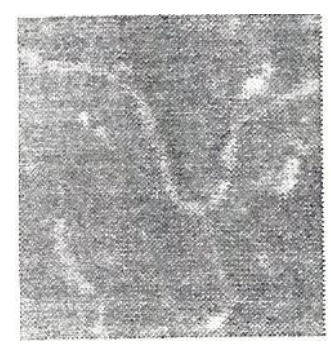

(a)Original image of 32 by 32 pixels of a portion of Landsat TM imagery data of Saga city in Kyushu, Japan

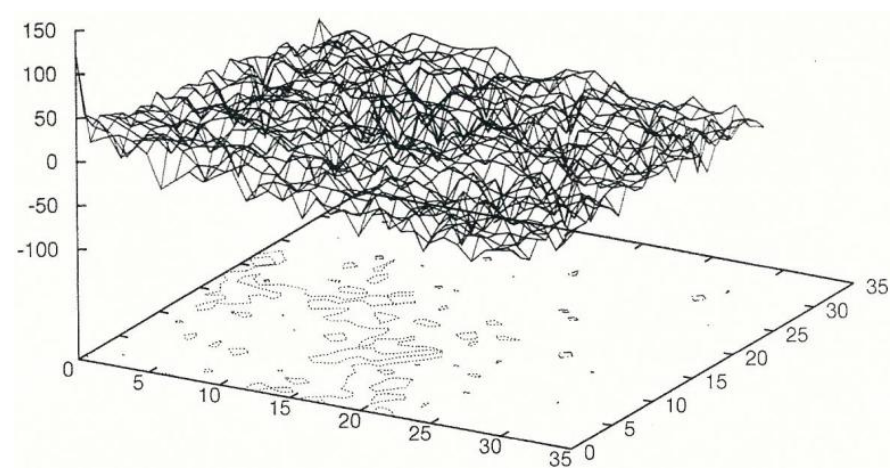

(b) Frequency component of the original image in direction of line and pixel

Fig. 1. Original image of 32 by 32 pixels of a portion of Landsat TM imagery data of Saga city in Kyushu, Japan and its 2D spectrum distribution.

\section{B. Effect of Median Filter}

In order to eliminate isolated noise pixels which are contained in the original image, median filter is applied to the original image. The resultant image is shown in Fig. 2 together with its frequency component in direction of line and pixel. 2D spectrum of Fig.2 (b) shows how the isolated noise pixels are eliminated maintaining major frequency components. 


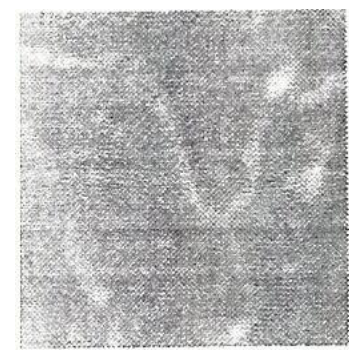

(a)Resultant image after applying median filter to the original image of 32 by 32 pixels of a portion of Landsat TM imagery data of Saga city in Kyushu, Japan

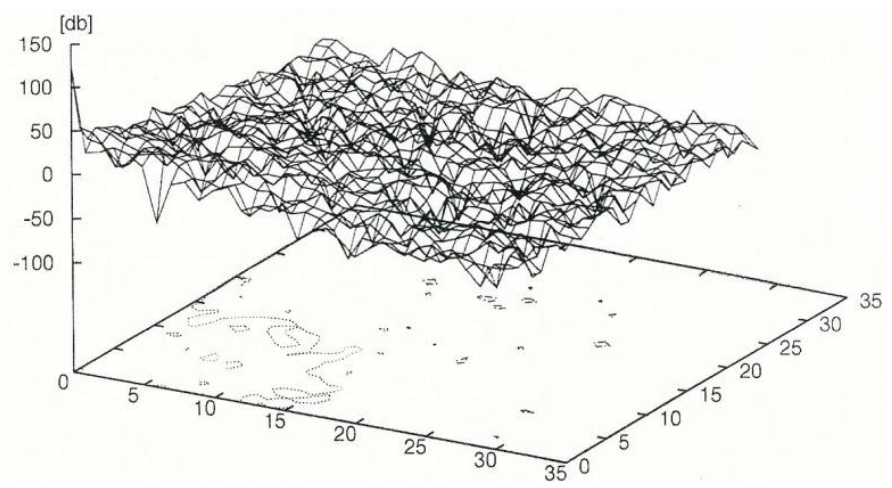

(b) Frequency component of the resultant image in direction of line and pixel

Fig. 2. Resultant image after applying median filter to the original image of 32 by 32 pixels of a portion of Landsat TM imagery data of Saga city in Kyushu, Japan and its 2D spectrum distribution.

\section{Effect of Additive Noise}

By using Mersenne Twister of random number generator [5], 30\% of averaged frequency component of the original image of Gaussian noise is added to the original image. Fig. 3 shows the noise added image together with frequency component in direction of line and pixel.

Also, Fig.4 shows the resultant image after applying median filter to the $30 \%$ of noise added original image of 32 by 32 pixels of a portion of Landsat TM imagery data of Saga city in Kyushu, Japan and its 2D spectrum distribution.

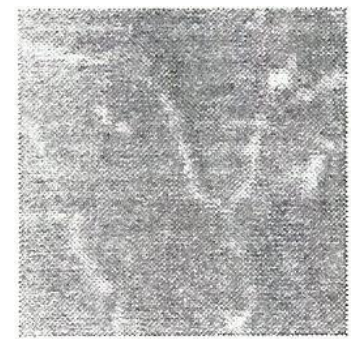

(a) $30 \%$ of noise added original image of 32 by 32 pixels of a portion of Landsat TM imagery data of Saga city in Kyushu, Japan

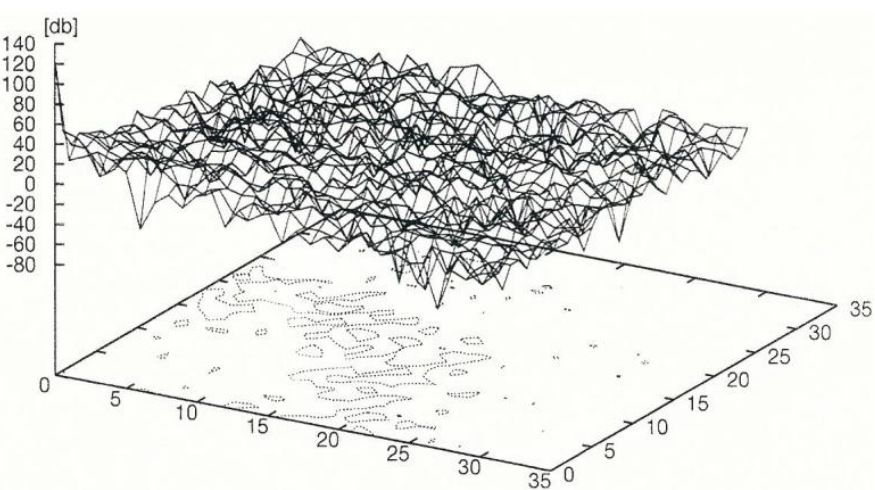

(b) Frequency component of the noise added image in direction of line and pixel

Fig. 3. $30 \%$ of noise added original image of 32 by 32 pixels of a portion of Landsat TM imagery data of Saga city in Kyushu, Japan and its 2D spectrum distribution.

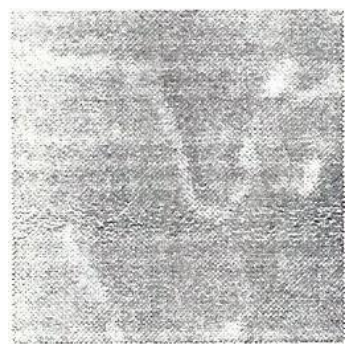

(a)Resultant image after apply median filter to the $30 \%$ of noise added original image of 32 by 32 pixels of a portion of Landsat TM imagery data of Saga city in Kyushu, Japan

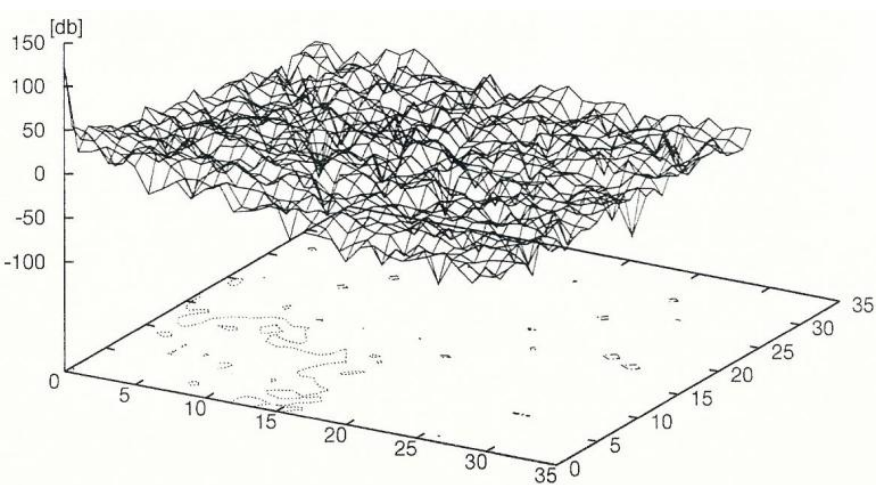

(b) Frequency component of the resultant image in direction of line and pixel

Fig. 4. Resultant image after applying median filter to the $30 \%$ of noise added original image of 32 by 32 pixels of a portion of Landsat TM imagery data of Saga city in Kyushu, Japan and its 2D spectrum distribution.

Meanwhile, Fig.5 shows $50 \%$ of noise added original image of 32 by 32 pixels of a portion of Landsat TM imagery data of Saga city in Kyushu, Japan and its 2D spectrum distribution.

On the other hand, Fig.6 shows the resultant image after applying median filter to the $50 \%$ of noise added original image of 32 by 32 pixels of a portion of Landsat TM imagery data of Saga city in Kyushu, Japan and its 2D spectrum distribution. From these Fig.4 and 6, it is found that median filter does work for isolated noise pixels. Also, higher frequency component increases in accordance with increasing added noise obviously. 


\section{Effect of the Proposed Edge Enhancement Method}

Using same imagery data, the proposed LOG filter based edge enhancement method based on GA is evaluated. In the experiments, cross-over probability is set at 0.21 and mutation probability is set at 0.05 . These parameters for GA are found empirically.

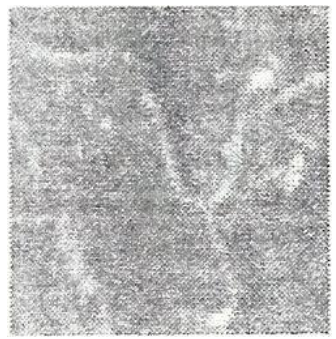

(a) $50 \%$ of noise added original image of 32 by 32 pixels of a portion of Landsat TM imagery data of Saga city in Kyushu, Japan

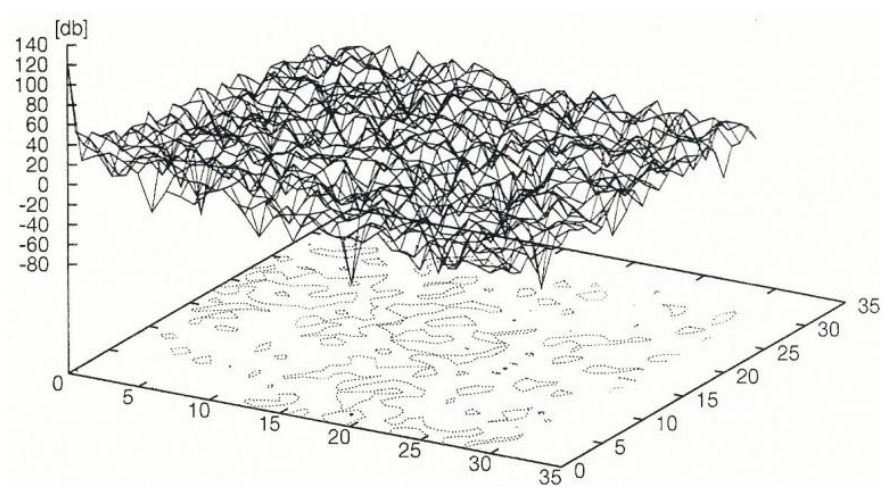

(b) Frequency component of the noise added image in direction of line and pixel

Fig. 5. $50 \%$ of noise added original image of 32 by 32 pixels of a portion of Landsat TM imagery data of Saga city in Kyushu, Japan and its 2D spectrum distribution.

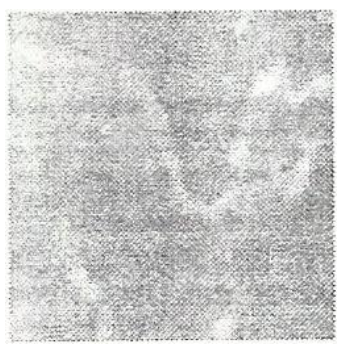

(a) Resultant image after applying median filter to the $50 \%$ of noise added original image of 32 by 32 pixels of a portion of Landsat TM imagery data of Saga city in Kyushu, Japan

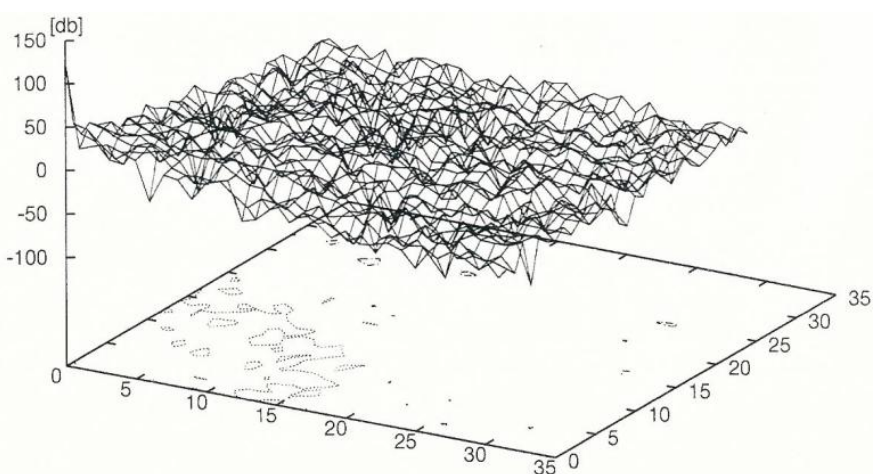

(b) Frequency component of the resultant image in direction of line and pixel

Fig. 6. Resultant image after applying median filter to the $50 \%$ of noise added original image of 32 by 32 pixels of a portion of Landsat TM imagery data of Saga city in Kyushu, Japan and its 2D spectrum distribution.

Fig. 7 and 8 shows the resultant images after applying the proposed method to $30 \%$ and $50 \%$ of noise added original image and their 2D spectra, respectively. Through comparison between Fig.7, Fig.8 and Fig.3, Fig.5, respectively, it is found that the proposed edge enhancement method does work for enhancing edges depending on the complexity of the image in concern with noise suppression.

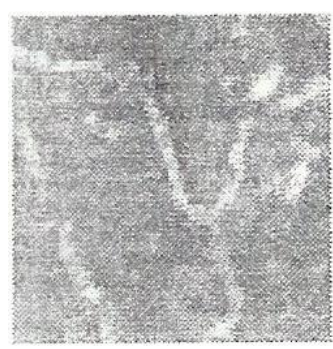

(a) Resultant image after applying the proposed edge enhancement method based on GA to the $30 \%$ of noise added original image of 32 by 32 pixels of a portion of Landsat TM imagery data of Saga city in Kyushu, Japan

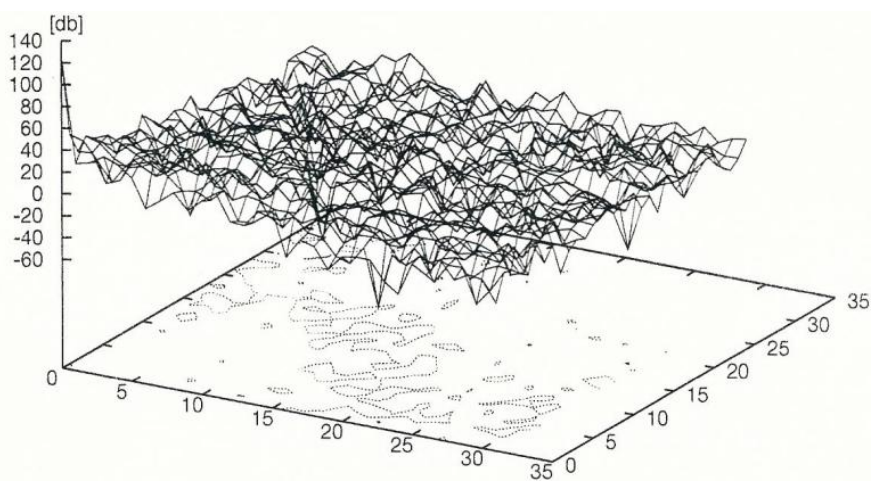

(b) Frequency component of the resultant image in direction of line and pixel

Fig. 7. Resultant image after applying the proposed edge enhancement method based on GA to the $30 \%$ of noise added original image of 32 by 32 pixels of a portion of Landsat TM imagery data of Saga city in Kyushu, Japan and its 2D spectrum distribution. 


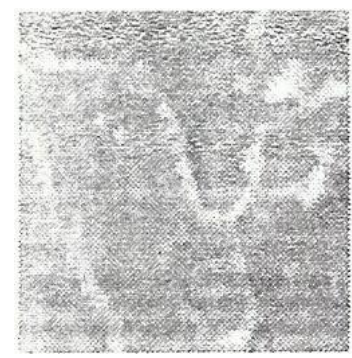

(a) Resultant image after applying the proposed edge enhancement method based on GA to the $50 \%$ of noise added original image of 32 by 32 pixels of a portion of Landsat TM imagery data of Saga city in Kyushu, Japan

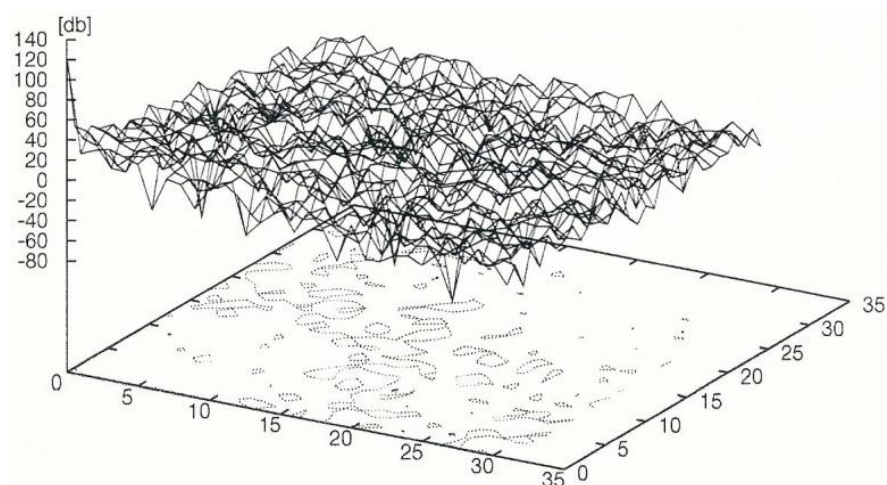

(b) Frequency component of the resultant image in direction of line and pixel

Fig. 8. Resultant image after applying the proposed edge enhancement method based on GA to the $50 \%$ of noise added original image of 32 by 32 pixels of a portion of Landsat TM imagery data of Saga city in Kyushu, Japan and its 2D spectrum distribution.

\section{E. Comparison to the Edge Enhancement Method Based on $S A$}

Using same imagery data, the proposed LOG filter based edge enhancement method based on GA is compared to the edge enhancement method based on SA. Fig.9 and 10 shows the resultant images after applying the SA based method to $30 \%$ and $50 \%$ of noise added original image and their $2 \mathrm{D}$ spectra, respectively. Through comparison between Fig.9, Fig.10 and Fig.7, Fig.8, respectively, it is found that the resultant images of the proposed edge enhancement method are almost same as the resultant images derived from the SA based edge enhancement method.

Both GA based and SA based edge enhancement methods work well for enhancing edges depending on the complexity of the image in concern with noise suppression. The number of iteration of GA is 164670 while that of SA is 8783578 . These numbers are found empirically with the convergence condition of residual error of 5\%. Namely, the proposed edge enhancement method is 53.34 times faster than the SA based method with almost same edge enhancement performance.

In comparison between the proposed edge enhancement method and median filter method, the resultant images after applying median filter show that it does not work for edge enhancement while noise suppression performance is good though. On the other hand, the proposed GA based edge enhancement method does work for edge enhancement with noise suppressing performance.

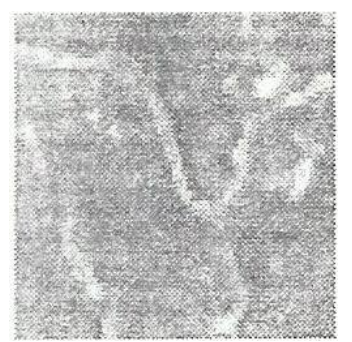

(a) Resultant image after applying the proposed edge enhancement method based on SA to the $30 \%$ of noise added original image of 32 by 32 pixels of a portion of Landsat TM imagery data of Saga city in Kyushu, Japan

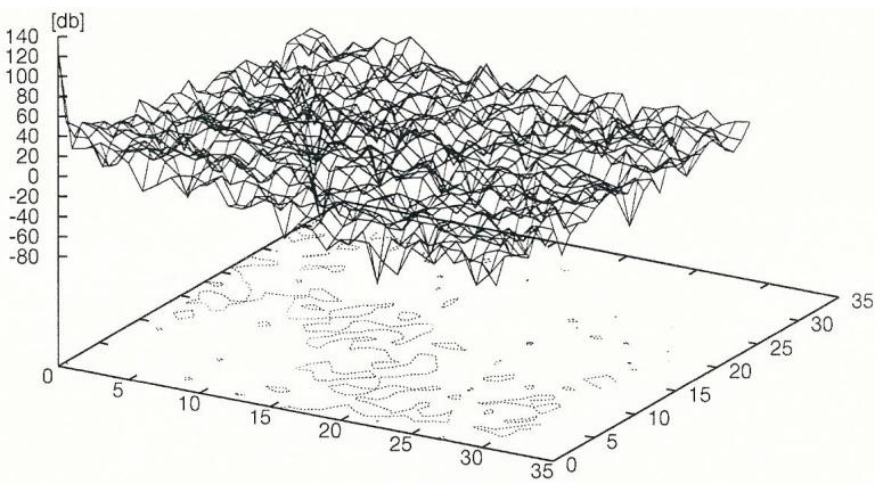

(b) Frequency component in direction of line and pixel

Fig. 9. Resultant image after applying the proposed edge enhancement method based on SA to the $30 \%$ of noise added original image of 32 by 32 pixels of a portion of Landsat TM imagery data of Saga city in Kyushu, Japan and its 2D spectrum distribution.

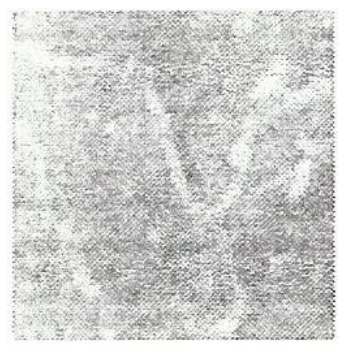

(a) Resultant image after applying the proposed edge enhancement method based on SA to the $50 \%$ of noise added original image of 32 by 32 pixels of a portion of Landsat TM imagery data of Saga city in Kyushu, Japan

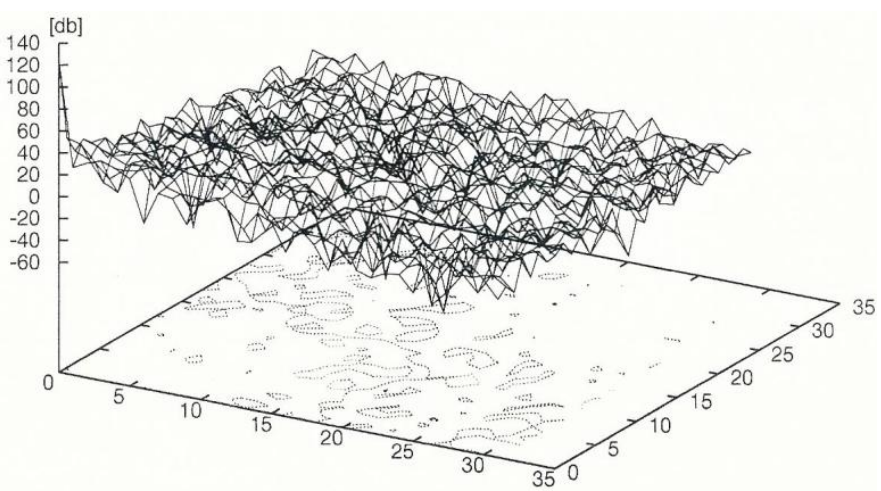

(b) Frequency component in direction of line and pixel

Fig. 10. Resultant image after applying the proposed edge enhancement method based on SA to the $50 \%$ of noise added original image of 32 by 32 pixels of a portion of Landsat TM imagery data of Saga city in Kyushu, Japan and its 2D spectrum distribution. 


\section{CONCLUSION}

LOG filter based method for noise suppressing edge enhancement based on Genetic Algorithm: GA taking into account complexity of target images measured with fractal dimension is proposed. Through experiments with satellite remote sensing imagery data with additive noise, it is found that the proposed method shows appropriate edge enhancing performance with suppressing the additive noise in accordance with complexity of target images. It is also found that the proposed method requires a small computer resources in comparison to the method based on Simulated Annealing: SA.

Through experiments with Landsat TM imagery data of Saga city, Kyushu, Japan, it is found that the proposed GA based edge enhancement method does work for edge enhancement depending on complexity of image with a good noise suppressing performance. The number of iteration of GA is 164670 while that of SA is 8783578 . These numbers are found empirically with the convergence condition of residual error of 5\%. Namely, the proposed edge enhancement method is 53.34 times faster than the SA based method with almost same edge enhancement performance.

\section{ACKNOWLEDGMENT}

The author would like to thank Mr. Mitsuki Nakamura for his efforts through experiments and simulations.

\section{REFERENCES}

[1] Arai, K., Lecture note for image processing, Gakujutsu-Tosho-Shuppan publicing Co. Ltd., 1999.

[2] Arai, K., Remote Sensing Satellite image processing methods with Java programing language, Morikita Shuppan publicing Co. Ltd., 2001.

[3] Arai, K., Fundamental theory for pattern recognition, Gakujutsu Tosho Shuppan, Publicing Co. Ltd., 1999.

[4] Arai, K, Lecture Notes on Remote Sensing, Morikita-Shuppan, Co.Ltd., 2005

[5] Mersenne Twister (MT), http://www.math.sci.hiroshima-u.ac.jp/ m$\mathrm{mat} / \mathrm{MT} / \mathrm{mt} . \mathrm{html}$

\section{AUTHORS PROFILE}

Kohei Arai, He received BS, MS and PhD degrees in 1972, 1974 and 1982, respectively. He was with The Institute for Industrial Science and Technology of the University of Tokyo from April 1974 to December 1978 also was with National Space Development Agency of Japan from January, 1979 to March, 1990. During from 1985 to 1987, he was with Canada Centre for Remote Sensing as a Post Doctoral Fellow of National Science and Engineering Research Council of Canada. He moved to Saga University as a Professor in Department of Information Science on April 1990. He was a councilor for the Aeronautics and Space related to the Technology Committee of the Ministry of Science and Technology during from 1998 to 2000. He was a councilor of Saga University for 2002 and 2003. He also was an executive councilor for the Remote Sensing Society of Japan for 2003 to 2005 . He is an Adjunct Professor of University of Arizona, USA since 1998. He also is Vice Chairman of the Commission "A" of ICSU/COSPAR since 2008. He wrote 30 books and published 322 journal papers 